\title{
Effect of Channel Coding With Node Cooperation for Wireless Passive Sensor Networks
}

\author{
${ }^{1}$ FarmanUllah, ${ }^{2}$ Aamir \\ Khan \\ ${ }^{1,2}$ COMSATS Insititute of IT \\ Quaid Avenue, WahCantt. \\ Pujnab, Pakistan
}

\author{
Hasan Farooq \\ UniversitiTechnologiPetronas, \\ Malaysia
}

\author{
Imdad Ullah \\ SEECS, NUST, Islamabad, \\ Pakistan
}

\begin{abstract}
The Passive sensor nodes are operated in a very low power regime i.e. $-10 \mathrm{dBm}$ to $-30 \mathrm{dBm}$. Due to this fact, there is a high chance of data to be lost or severely corrupted due to overcome of noisy environment. In order to tackle this problem it was suggested that node cooperation is able to combat this. Node cooperation is very helpful in order to take the data node by node until to the final destination, rather to send directly from source to destination. This work is achieved by first modelling an empirical system consists of single relay, source and destination. And the two relaying protocols (SF and DF) were modelled and implemented. This approach is then extended for three relay nodes and the two sets of relaying nodes were implemented again on every single node. The output performances were compared, and further improvement was seen by channel coding.
\end{abstract}

Keywords: Wireless Passive Sensor Networks, Node Co-

operation, Channel Coding

\section{INTRODUCTION}

In wireless sensor networks, signal is severely degraded by the multipath signals which can be compensated with the use of diversity. Taking the advantageous of diversity, the destination node accumulates or combines all the received signals received via independent links and makes the final decision upon the transmitted bits. This characteristic of sensor networks is called the cooperative attempt and is always there when they are active. Sensor nodes, with this property enhance their quality of service and offer good BER compare to the conventional mode of communication. The senor nodes make use of their processing capabilities to locally carry out simple calculations and send out only interested data [5]. This approach or capability of WPSN can offer spatial diversity against fading in a wireless channel4 [22]-[23]. If there is no direct line of sight or having difficulty in communication, then one sensor node help another, called relay node in order to accomplish transmission from source to the desired destination [23]. This cooperative behaviour is adopted by each sensor node in the network along with the normal communication responsibility.Node Cooperation results in various trades off in terms of resources e.g. data rate and transmit power. Because in cooperative mode if there are " $\mathrm{N}$ " number of sensor nodes, then the total power would be divided into "N" number of portions and with that individual part of the total power, the data would be transmitted to each node. Hence, total power available at the single node divided and thus it is reduced for all users because of diversity [24].The scenario that this work will consider would assume that all nodes work in the same band and therefore the source node will behave in broadcast manner, while the destination node is in multiple channel access modes as depicted in the following figure 1. Channel between each pair of node is independent and have random effect on data.

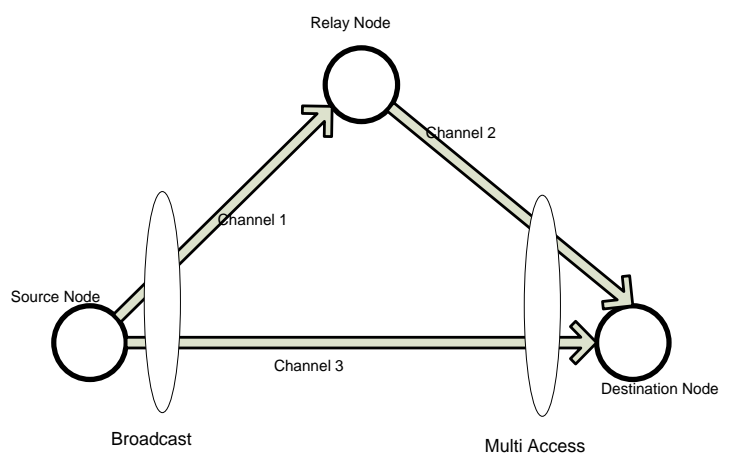

Fig 1: A 2 hops relaying system

Cooperative behaviour of sensor nodes created new approaches to enhance the sensor lifetime, low cost and multi functional operation in the sensor field. This approach created spatial diversity against fading in a wireless channel. In the case of sever fading channel, the source node is assisted by the relay channel to reach the information to the destination. This is basically done by the node relaying protocol called AF and DF. If the received signal SNR is below the threshold, due to which transmission is made unsuccessful, therefore message is encoded, re-encoded and retransmitted towards the destination. This decision is taken by the relay node on the single bit feedback, broadcasted by the destination that is at least reliably received by the relay node. Information is either corrupted by Reflection, Scattering or Diffraction etc. or due to the Small Scale Fading effect of the channel.

\section{SYSTEM MODEL, APPROACH AND ACHIEVED RESULTS WITH DIS- CUSSION}

When signal is transmitted over a long communication path, the received signal is observed in a different form than the original. The envelope amplitude of this signal when observed would seem to be fluctuating as the time passes by. This effect is caused by "fading". This effect puts some limitations on the system design. However, if different signals are received, then fading over one path is different than another [34],[35]. Therefore if copy of the same signal is received via different links, then it is rarely happen that all channels are severely faded but it is good for reducing fading effect. This technique is called "diversity" which improves the combined signal to noise ratio and process of combining these signals at the receiver is called "Maximal Ratio Diversity" or Maximum 
SYSTEM DESIGN:Data passed through each relay node would be passed through the process shown by the following block diagram but only if the serving relay is choosing DF mode for retransmission of the received signal.

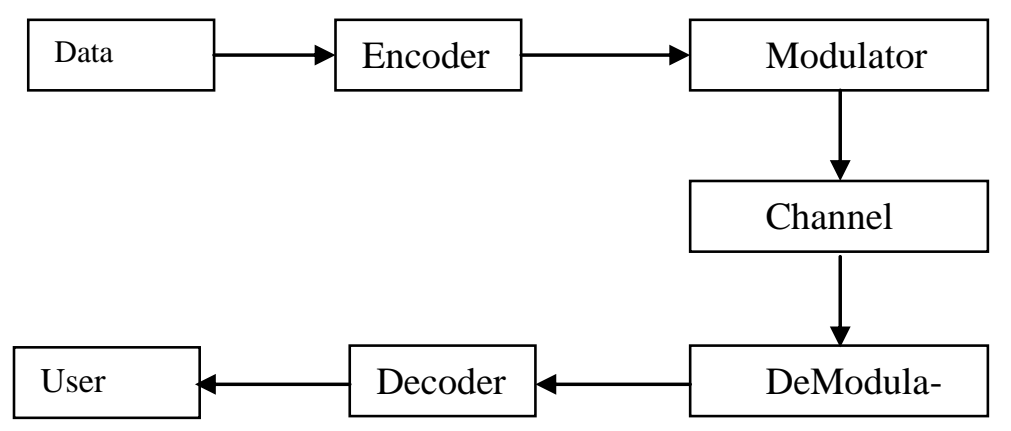

Fig 2: Block diagram of a Digital Communication Process System

Block diagram shows that initially, the data source randomly generates bit stream of $0 \mathrm{~s}$ and $1 \mathrm{~s}$. Data stream is passed through encoder. Half convolutional encoder would be used in system design, which will introduce redundant bits equal to the information bits i.e. each information bit would have one redundant bit. The encoded bits would be modulated using different modulation schemes e.g. BPSK, QPSK etc. The modulator would generate a set of finite time duration wave-

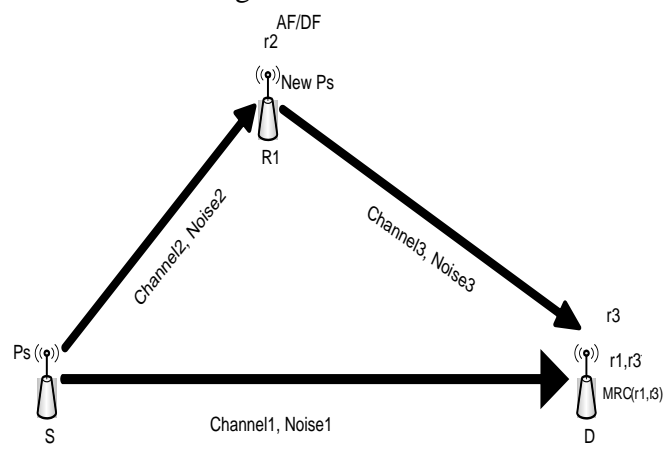

Fig

3: One Relaying node using either AF or DF

Results-1: One Relay Coded system using BPSK, QPSK and 8PSK schemes

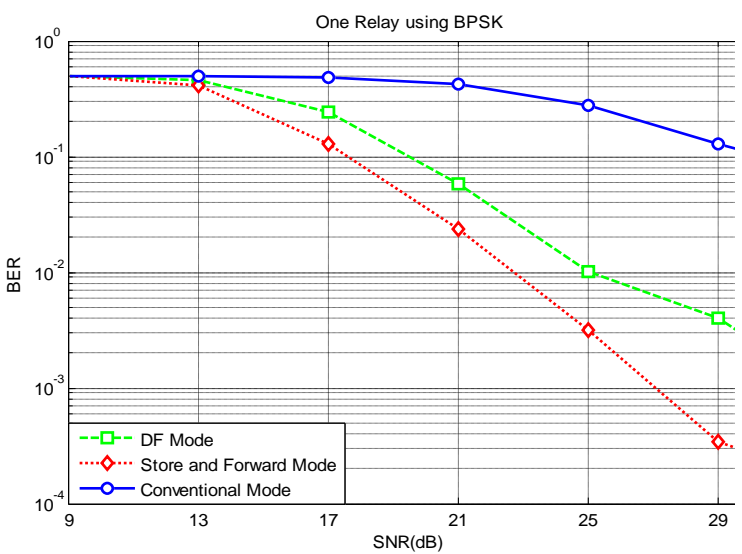

Fig 4: One Relay outputs using BPSK

One Relay output using BPSK. The unity power is shared between relay and the destination node i.e. source transmits data towards relay and destination with half power. forms and will provide mapping between the encoder output and the generated set of waveforms. The modulated signal is passed through flat fade channel and channel state information is known at the receiver. Channel introduces multiplicative noise but at the receiver, the received signal is also distorted by the AWGN noise. At the receiver, the same modulation scheme is used to de-map the signal and user data is decoded from the de-mapped signal.

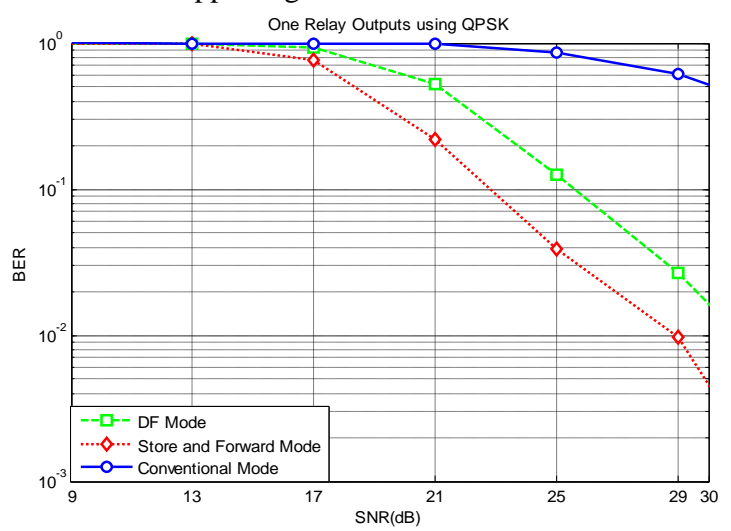

Fig 5: One Relay outputs using QPSK

One Relay output using QPSK. The unity power is shared between relay and the destination node i.e. source transmits data towards relay and destination with half power.

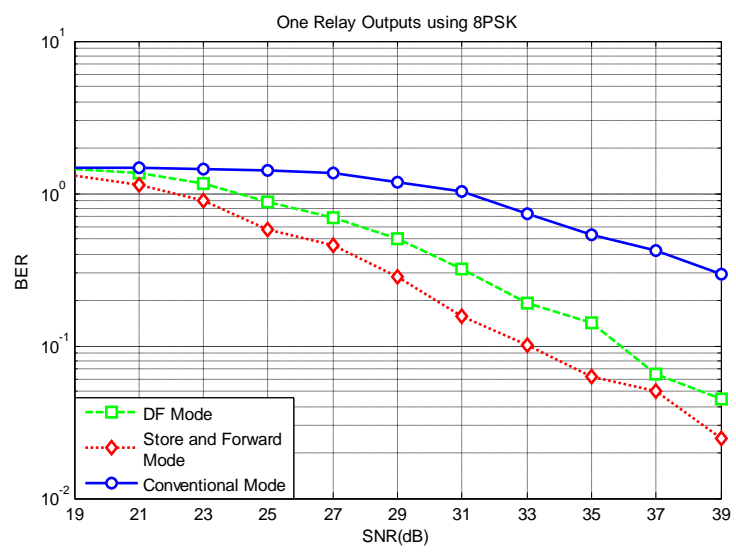

Fig 6: One Relay outputs using 8PSK 
One Relay output using 8PSK. The unity power is shared between relay and the destination node i.e. source transmits data towards relay and destination with half power.

Discussion of Results-1:Figure 4-6, show the bit error probability of three types of transmission mode over a range of SNR. These are the outputs of a coded system using $1 / 2$ rate convolutional code. The performance is observed over three types of different modulation schemes and throughout it is found that $\mathrm{AF}$ takes full advantage in terms of providing less transmission error compare to other two.

But there is obvious change in error probability when the number of sensor nodes increased. The reason is that, each sensor node has now more choices to achieve path diversity and to transfer the desired data over various paths. This offers more capability to combat fading and achieve.

When number of relay nodes increase, the serving node will equally distribute the total available power among all relay nodes and with that fraction of power, the data would be sent to each relaying node including destination node.

Power consumption should be at minimum if there are more relays available to help the serving node. This statement will also be proved at the end of this section. Moreover for comprehensive results, it is important to extend the model for multiple relaying nodes. The above model is for single relay, while in practical scenarios, a network consists of many relay nodes. Therefore, model is extended for multiple relays as depicted by the following diagram.

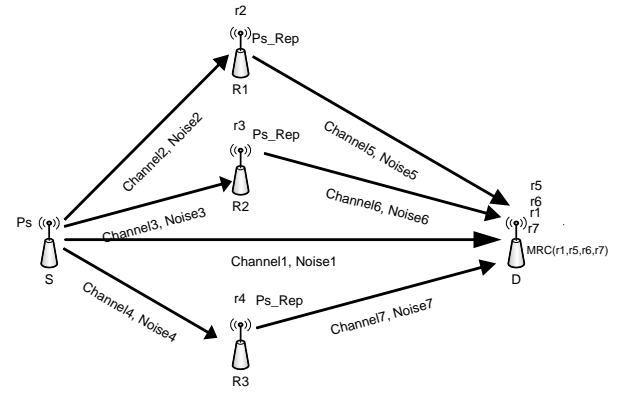

Fig 7: Diversity in Multiple nodes using Repeater Mode

In Figure 7, it is only one transmit antenna and 4 receive antennas. Each receive antenna has a distinct and independent experience of channel effect. The received data ( $\mathrm{r} 2, \mathrm{r} 3$ and $\mathrm{r} 4)$

Substituting the values in equation 3 at relay R1, R2 and R3 is amplified by the corresponding power factors which are introduced by each relay node distinctly.

At the destination, these are combined to evaluate the resultant SNR. Since each relay simply amplifies what they receive, therefore at the destination node, each received data is having the channel effect through which the received data had passed. Therefore, received data is equalized at the destination and then the user data is extracted after decoding. Mathematical analysis for the repeater mode is as follow.

Suppose, Signals received by destination node and R1 as

$$
\begin{aligned}
& y_{S, D}=\sqrt{P_{S}} h_{S, D} S+\vartheta_{S, D} \\
& y_{S, R 1}=\sqrt{P_{S}} h_{S, R 1} S+\vartheta_{S, R 1}
\end{aligned}
$$

$y_{S, D}$ and $y_{S, R 1}$ correspond to $\mathrm{r} 1$ and $\mathrm{r} 2$ in above figure. Each received signal has flat fad channel effect denoted by $h_{S, D}$ and $h_{S, R 1}$ and is different from each other. Each transmitted data is multiplied with a randomly varying complex number $h_{S, D}$ and $h_{S, R 1}$. Since the channel considered in this model is Rayleigh channel, therefore real and imaginary parts are Gaussian distributed with "mean $=0$ " and "variance $=1 / 2$ ". Also the noises are distinct on each link and are uncorrelated with respect to the noise received by each antenna.

Destination nodes receives signal via relay as

$$
\begin{aligned}
& y_{R 1, D}=\sqrt{P_{R 1}}\left(y_{S, R 1}\right) h_{R 1, D}+\vartheta_{R 1, D} \\
& O R \\
& r 5=\sqrt{P_{R 1}}\left(\sqrt{P_{S}} h_{S, R 1} S+\vartheta_{S, R 1}\right) h_{R 1, D}+\vartheta_{R 1, D}
\end{aligned}
$$

Since the channel is known at the receive antenna, therefore the destination node equalizes the two received signals and combine them as

$$
M R C=\frac{y_{S, R 1}}{h_{S, R 1}}+\frac{y_{R 1, D}}{h_{S, R 1} * h_{R 1, D}}
$$

$$
\begin{aligned}
& =\sqrt{P_{S}} S+\frac{\vartheta_{S, R 1}}{h_{S, R 1}}+\frac{\sqrt{P_{R 1}}\left(\sqrt{P_{S}} h_{S, R 1} S+\vartheta_{S, R 1}\right)}{h_{S, R 1}}+\frac{\vartheta_{R 1, D}}{h_{S, R 1} * h_{R 1, D}} \\
& =\sqrt{P_{S}} S+\frac{\vartheta_{S, R 1}}{h_{S, R 1}}+\frac{\sqrt{P_{R 1}}\left(\sqrt{P_{S}} h_{S, R 1} S\right)}{h_{S, R 1}}+\frac{\sqrt{P_{R 1}} \vartheta_{S, R 1}}{h_{S, R 1}}+\frac{\vartheta_{R 1, D}}{h_{S, R 1} * h_{R 1, D}}
\end{aligned}
$$

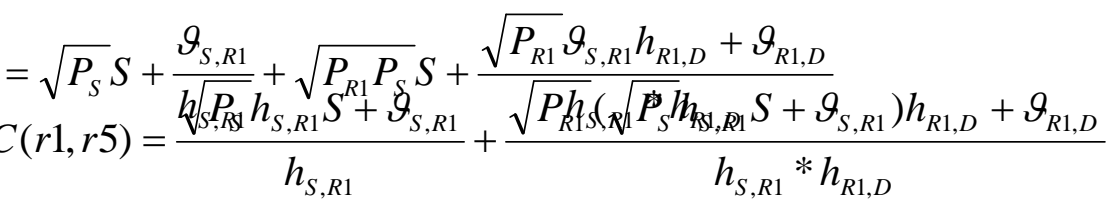

Rearranging the signal and noise part of the equation

$$
\underbrace{=\sqrt{P_{S}}}_{\text {Signal Pover }} S+\underbrace{\sqrt{P_{R 1} P_{S}} S+\frac{\vartheta_{S, R 1}}{h_{S, R 1}}+\frac{\sqrt{P_{R 1}} \vartheta_{S, R 1} h_{R 1, D}+\vartheta_{R 1, D}}{h_{S, R 1}} * h_{R 1, D}}_{\text {Noisetrower }}
$$


This is the combined signal of $r 1$ and $r 5$. However each signal passed through each relay path could be worked out in the same fashion.

At the destination node, the received signal has the signal and noise power as shown in equation 4 . The ratio of the expectation of signal power to the expectation of noise power gives SNR and could be written as.

$$
\begin{aligned}
& S N R=\frac{\zeta\left\{\sqrt{P_{S}} S+\sqrt{P_{R 1} P_{S}} S\right\}^{2}}{\zeta\left\{\frac{\vartheta_{S, R 1}}{h_{S, R 1}}+\frac{\vartheta_{S, R 1}+\vartheta_{R 1, D}}{h_{S, R 1} * h_{R 1, D}}\right\}} \\
& S N R=\frac{P_{S} \delta^{2}+P_{R 1} P_{S} \delta^{2}}{N_{0}}
\end{aligned}
$$

In our system model since various SNR would be applied to see the error performance; the performance of which could be seen from equation above. As the SNR increases, the noise factor is dominated by the signal factor and thus error probability is expected to be decreased constantly.

Each relay also has the choice to select the DF mode for retransmitting the received message as it could be seen in the following diagram.

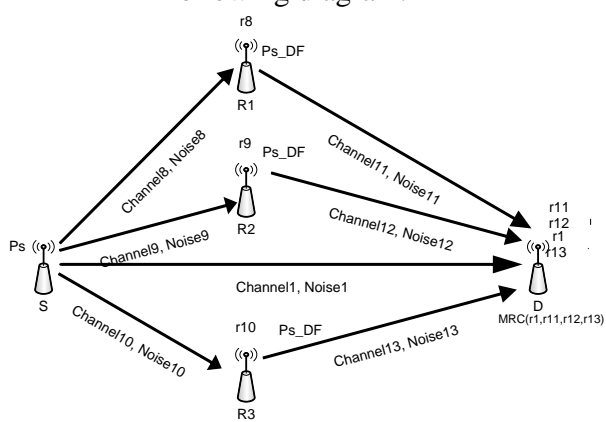

Fig 8: DF Mode Diversity in Multiple nodes

Each relay in above diagram first decodes the signal. The decoded bits are retransmitted via the distinct channel to the final destination. At the destination node, four uncorrelated signals are received and equalized individually. The equalized signals are combined using MRC to evaluate the resultant SNR. Mathematical analysis for the DF mode is as follow.

In a most simple case, suppose the destination node in figure 8 receives two uncorrelated signals $r 1$ and $r 11$ e.g.

$$
\begin{aligned}
& r 1=y_{S, D}=\sqrt{P_{S}} h_{S, D} S+\vartheta_{S, D} \\
& r 11=y_{R 1, D}=\sqrt{P_{P_{S} D F}} h_{R 1, D} \hat{S}+\vartheta_{R 1, D}
\end{aligned}
$$

Where $S$ are the information bits decoded by the relay node in advance. The relay node retransmits these bits with a new power factor represented by "Ps_DF". The equalized signals are combined at the destination node such as.

$$
\begin{aligned}
\operatorname{MRC}(r 1, r 11)=\frac{y_{S, D}}{h_{S, D}}+\frac{y_{R 1, D}}{h_{R 1, D}} & \\
= & \frac{\sqrt{P_{S}} h_{S, D} S+\vartheta_{S, D}}{h_{S, D}}+\frac{\sqrt{P_{P_{S} D F}} h_{R 1, D} \hat{S}+\vartheta_{R 1, D}}{h_{R 1, D}}
\end{aligned}
$$

$$
=\sqrt{P_{S}} S+\frac{\vartheta_{S, D}}{h_{S, D}}+\sqrt{P_{P s_{-} D F}} \hat{S}+\frac{\vartheta_{R 1, D}}{h_{R 1, D}}
$$

Re-arranging the signal and noise part of the equation 7

$$
\underset{2.6)}{\operatorname{MR} C}(r 1, r 11)=\sqrt{P_{S}} S+\sqrt{P_{P_{S_{-}} D F}} \hat{S}+\frac{\vartheta_{S, D}}{h_{S, D}}+\frac{\vartheta_{R 1, D}}{h_{R 1, D}}
$$

$$
\begin{array}{r}
S N R=\frac{\zeta\left\{\sqrt{P_{S} S}+\sqrt{P_{R 1}} S\right\}^{2}}{\zeta\left\{\frac{\vartheta_{S, D}}{h_{S, D}}+\frac{\vartheta_{R 1, D}}{h_{R 1, D}}\right\}} \\
S N R=\frac{P_{S} \delta_{S}{ }^{2}+P_{R 1} \delta_{R 1}{ }^{2}}{N_{0}}
\end{array}
$$

Results-2: 3 Relays Un-coded system with BPSK, QPSK and 8PSK

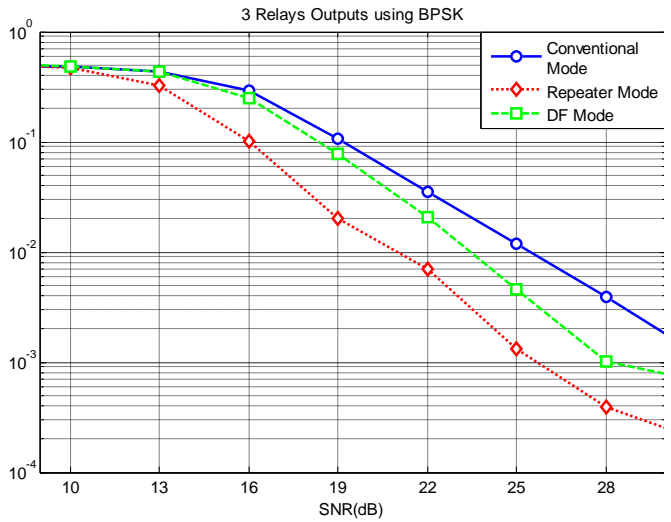

Fig 9: Three Relays outputs using BPSK

Three Relays outputs using BPSK. The unity power is shared among three relays and the destination node i.e. source transmits data towards relays and destination with one fourth power.

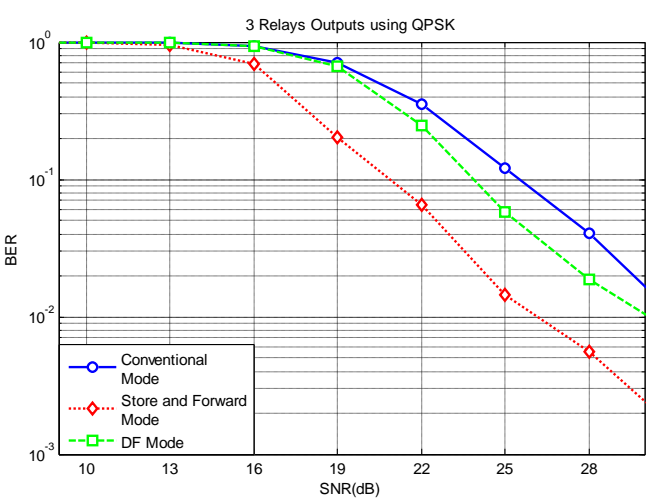

Fig 10: Three Relays outputs using QPSK 
Three Relays outputs using QPSK. The unity power is shared among three relays and the destination node i.e. source transmits data towards relays and destination with one fourth power.

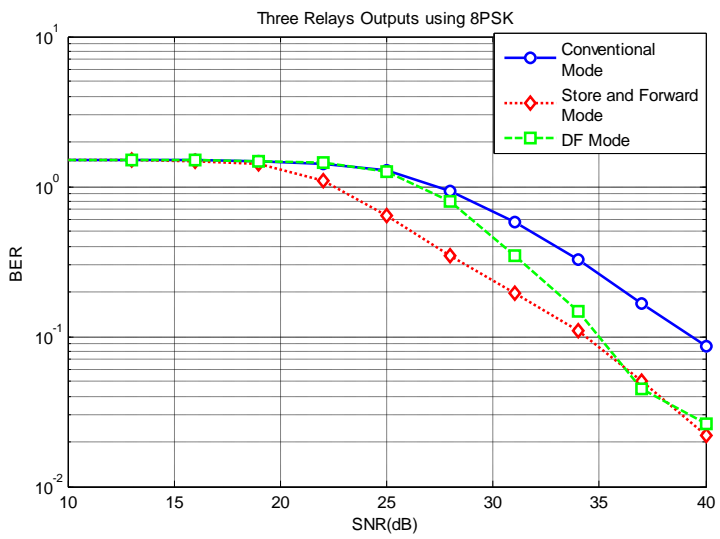

Fig 11: Three Relays outputs using 8PSK

Three Relays outputs using 8PSK. The unity power is shared among three relays and the destination node i.e. source transmits data towards relays and destination with one fourth power.

Discussion of Results-2: Results showing very obvious changes when number of relays are increased i.e. the error probability decreases when relaying nodes are changed from one relay to three. For example in BPSK the BER in One relay is slightly above from $10^{-3}$ at $28 \mathrm{~dB}$, while the same bit error rate is achieved at nearly $25 \mathrm{dBs}$ in the case of three relays.

This could be further improved by the channel coding as it could be seen from the following results.

Results-3: Channel Coding for 3 node relaying system using BPSK, QPSK and 8PSK.

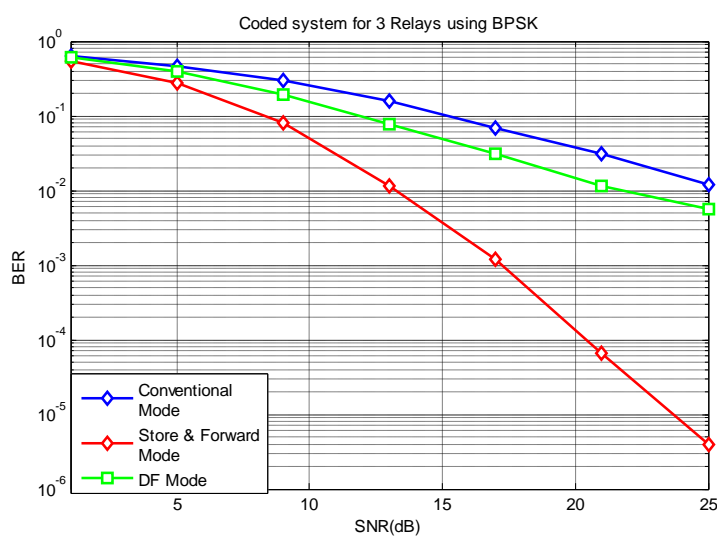

Fig 12: 3 Relays outputs using $1 / 2$ Convolutional Encoder

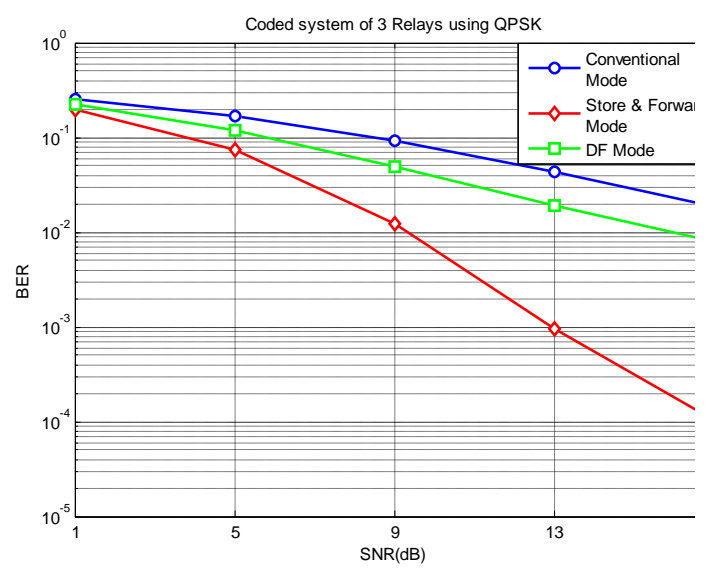

Fig 13: Coded system of 3 relaying nodes

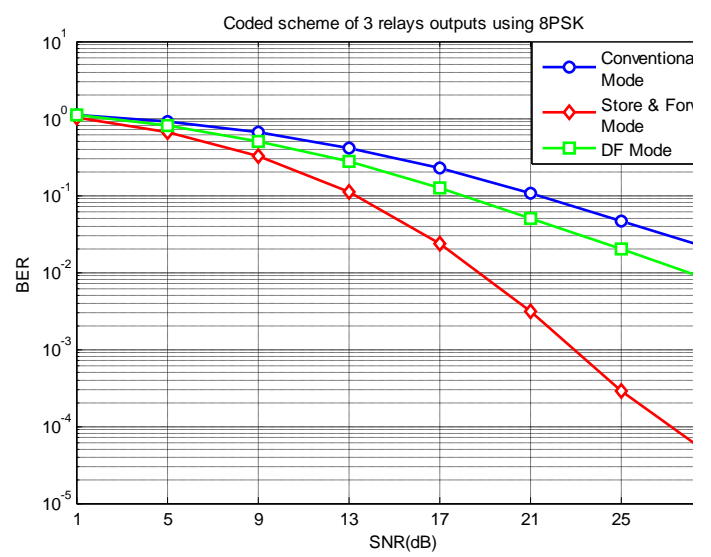

Fig 14: Coded system of 3 relaying nodes using 8PSK

Discussion of Results-3: Results were improved by the use of channel coding. Comparing figure 9 and 12, the same BER of $10^{-3}$ is achieved at $26 \mathrm{~dB}$ in un-coded system, while in coded system; this is achieved at nearly $17 \mathrm{~dB}$ SNR.

Results-4: 3 Relays outputs transmit data with total available power.

The system performance was observed to be improved further when transmitted the received data with maximum power. If compare the results of figure 9 with 15 , it would be found that error probability reduces nearly 1.5 times with full transmit power. The results with different modulation schemes could be observed from the following figure 15, 16 and 17.

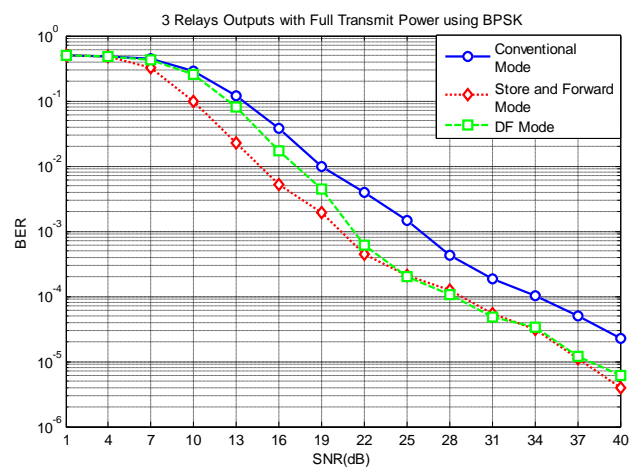

Fig 15: 3 Relays transmission with total available power using BPSK 


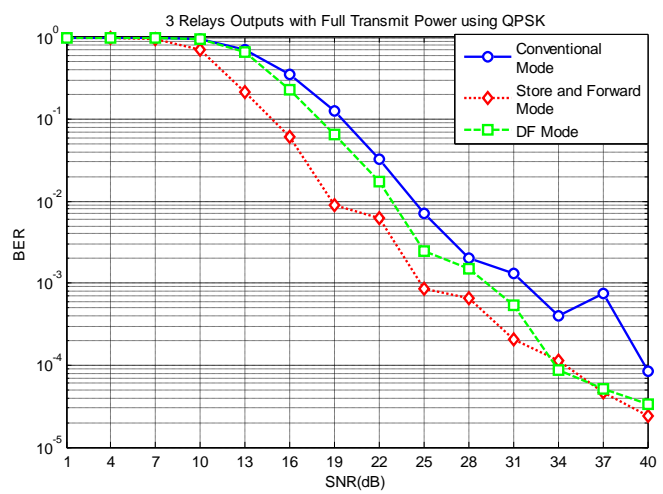

Fig 16: 3 Relays transmission with total available power using QPSK

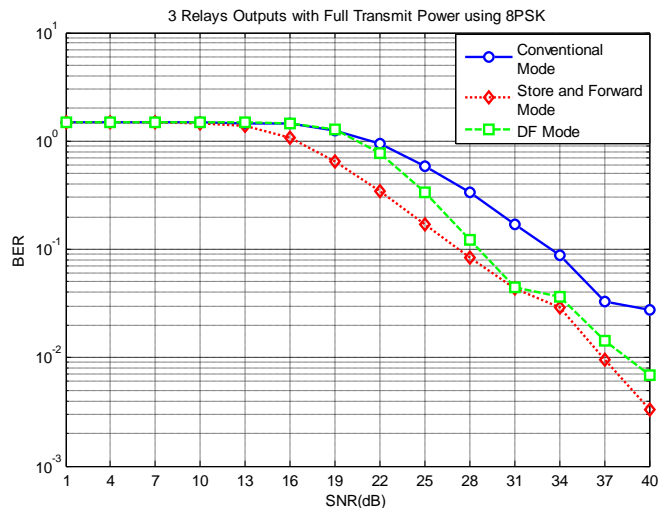

Fig 17: 3 Relays transmission with total available power using BPSK

\section{CONCLUSION}

The cooperative system performance was investigated in chapter4 for two types of communication e.g. Cooperative communication with two relaying protocols and direct communication between source and destination. First of all, it has been proved from all graphs, the cooperative communication is always winning in multiple relays, no matter it is compared to $\mathrm{AF}$ or $\mathrm{DF}$ i.e. it provides low BER compare to uncooperative communication, which provided that cooperative transmission offers spatial diversity against fading. Also it is reported that channel coding further improves the efficiency.

\section{REFERENCES}

[1] Jennifer Yick, Biswanath Mukherjee, DipakGhosal, "Wireless Sensor Network Survey," J.Yick et al./ Computer Networks 52 (2008), pp.2292-2330, April 2008.

[2] X,-Y. Li; "Multicast Capacity of Wireless Ad Hoc Networks", IEEE/ACM Transaction on Networking, vol.7, no.13, pp.950-961, June2009.

[3] G,-K. Chang, Z. Jia, J. Yu. A. Chowdhury, T. Wang, G. Ellinas, "Super-Broadband Optical Wireless Access Technologies," OFC/NFOEC 2008.

[4] A. Bereketli and O. B. Akan, "Communication Coverage in Wireless Passive Sensor Networks," IEEE Comm. Letters, vol. 13, no. 2, pp. 133-135, Feb. 2009.

[5] I.F.Akyildiz, W.Su, Y.Sankarasubramaniam, E. Cayirci, "Wireless Sensor Networks: A Survey "Computer Networks 38 (2002), pp.393-422, Dec 2001.
[6] Alexander Becher, ZinaidaBenenson, and MaximillianDornseif, "Tampering with Motes: RealWorld Physical Attacks on Wireless Sensor Networks," pp. 1-15.

[7] Adrian Perrig, John Stankovic, David Wagner, "Security in wireless sensor networks,"Commun.ACM, 47(6), pp.53-57, 2004.

[8] Elaine Shi and Adrian Perrig, "Designing secure sensor networks," IEEE Wireless Communications, 11(6), pp.38-43, December 2004.

[9] O. B. Akan, M. T. Isik, B. Baykal, "Wireless Passive Sensor Networks," IEEE Comm. Magazine, vol. 47, no. 8, pp. 92-99, Aug. 2009.

[10] D. Sakamoto and H. Higaki "Wireless Multihop Transmission with Buffering in Neighbour Sensor Nodes for Short Delay," IEEE Communications Society, WCNC 2009.

[11] Shi Yongjie, Sun Guiling, Liu Bo. Li Weixiang, "Design of Wireless Sensor Network Node Based on C8051F020 and CC1100 Fiber Bragg Grating," IEEE, pp. 1-4.July 2010.

[12] C. Intanagonwiwat, R. Govindan, and D. Estrin, "Directed Diffusion: A Scalable and Robus Communication Paradigm for Sensor Networks," Proc. ACM MobiCom 'OO, Boston, MA, 2000, pp. 56-57.

[13] H. Stockman, "Communication by means of Reflected Power,” Proc. I.R.E., vol. 36, 1948, pp.1196-1204

[14] M. Kossel, H. R. Benedickter, R. Peter, Batchtold, "Microwave Backscatter Modulation Systems," in IEEE MIT-S Digest 2000, pp.1427-1430.

[15] C.Turner, "Backscatter modulation of Impedance Modulated RFID tags," pp. 1-5, Feb 2003.

[16] M. TalhaIsikOzgur B. Akan, "Padre: Modulated Backscattering-based Passive Data Retrieval in Wireless Sensor Networks," IEEE Comm So, WCN 2009 Proc,

[17] F. Kocer, P.M. Walsh, and M. P. Flynn. "Wireless Remotely Powered Telemetry in $0.25 \mathrm{~m}$ CMOS," Proc, IEEE RFIC, June 2004, pp. 339-342.

[18] Yanqiu Li, Hongyun Yu, Bo Su, and Yonghong Shang, "Hybrid Micropower Source for Wireless Sensor Network," IEEE Sensors Journal,Vol 8, N0.6,June 2008, pp. 678-681.

[19] A. K. Marath, Y. W. M. Chia, C. C. Ko, "Performance Analysis of a Homodyne Receiver in Modulated Backscattered System for Intelligent Transportation." IEEE 1999, pp. 1198-1202.

[20] K. Han, Y. Choi, S. Choi, and Y. Kwon, "Power Amplifier Characteristic aware energy efficient Transmission Strategy," LNCS, vol.4479, pp. 37-48, Springer, Nov.2007.

[21] Product Datasheet "P2110-915MHz RF Powerharvester Receiver,"PowerCast, Rev A-2010/04, pp.1-12.

[22] M. R. Souryal, B. R. Vojcic, "Performance of Amplifyand-Forward and Decode-and-Forward Relaying in Rayleigh Fading with turbo codes,'IEEE ICASSP, pp.IV68-IV-684,2006. 
[23] J. Lee, S. Kim, H. S. T. Kwon, Y. Choi, J. S and A. Par, "Downlink Node Cooperation with Node Selection Diversity," IEEE, 2005.

[24] N. Laneman, D. N. C. Tse, G. W. Wornell, "Cooperative Diversity in Wireless Networks Efficient Protocols and Outage Behavior," IEEE Trans: on infor theory, vol,50, No, 12, pp. 2062-3080, 12 Dec 2004.

[25] A. Nosratinia, T. E. Hunter, A. Hedayat, "Cooperative Communication Wireless Networks," Adaptive Antennas and Mimo Systems for Wireless Comm, IEEE Comm: Magazine, pp. 74-80, Oct 2004.

[26] N. Laneman, D. N. C. Tse, G. W. Wornell, "Cooperative Diversity in Wireless Networks Efficient Protocols and Outage Behavior," IEEE Trans: on infor theory, vol,50, No, 12, pp. 2062-3080, 12 Dec 2004.

[27] J. G. Proakis, Digital Communication, $4^{\text {th }}$ ed. New York: McGraw-Hill, Inc., 2001

[28] T. M. Cover and A. A. El Gamal, "Capacity theorems for the relay channel," IEEE Trans. Infor, Theory, vol. IT-25, pp.572-584, Sept.1979.

[29] M. Yu and J. Li, "Is amplify-and-forward practically better than decode-and-forward or vice versa?",Proc. IEEE Int. Conf. Acoustics, Speech, and Signal Processing (ICASSP). Vol. 3, pp.365-368. March 2005.

[30] M-S. Alouini, A. Goldsmith, "Capacity of Rayleigh Fading Channels Under Different Adaptive Transmission and Diversity-Combining Techniques," IEEE Trans: on Vehicular Tech:, Vol:48, No. 4, pp. 1165-1181, July 1999.

[31] T. Sunaga, S. Sampei, "Performance of multi-level QAM with post-detection maximal ration combining space diversity of digital land-mobile radio communications," IEEE Trans: Vehicular Technology, Vol. 42, pp.294-301, Aug 1993.

[32] J. N. Laneman, "Cooperative Diversity in Wireless Networks: Algorithms and Architectures," Ph.D. dissertation, Massachusetts Institute of Technology, Cambridge, MA, Aug. 2002.
[33] X. Bao, J. LI, "Efficient Message Relaying for Wireless User Cooperation: Decode-Amplify-Forward (DAF) and Hybrid DAF and Coded-Cooperation," IEEE Trans: on Wireless Comm:, Vol. 6, pp. 3975-3985, Nov. 2007.

[34] T. Q. Duong, H-J.Zepernick, "Hybrid Decode-AmplifyForward Cooperative Communications with Multiple Relays," IEEE Comm: Soc: WCN 2009 Proc:,pp. 1-6

[35] C. R. C. M. Da Silva, M.D Yacoub, "A Generalized Solution for Diversity Combining Techniques in Fading Channels," IEEE Trans: on Microwave Theory and Techniques, Vol. 50, No.1, pp. 46-50, Jan 2002.

[36] B.S. Paul, R. Bhattacharjee, "Maximal ratio combining of two amplify-forward relay branches with individual 1 inks experiencing Nakagami fading," IEEE, pp.1-4, 2007.

[37] A. Sendonaris, E. Erkip, B. Aazhang, "User Cooperation diversity-part-1: system description," IEEE Transactions on Comm: Vol. 51, no.11, Nov 2003.

[38] A. Sendonaris, E. Erkip, B. Aazhang, "User Cooperation diversity-part-II: Implementation aspects and performance analysis," IEEE Transactions on Comm:, vol.51, no.11, Nov 2003

[39] Product Datasheet "P2110-915MHz RF Powerharvester Receiver,"PowerCast, Rev A-2010/04, pp.1-12.

[40] http://www.rfwirelesssensors.com/2010/04/powercastp2110-battery-free-wireless-sensor-node-2010-04-30/

[41] G. Hoblos, M.Staroswiecki, and A.Aitouche, "Optimal Design of Fault Tolerant Sensor Networks," IEEE Int'l.Conf. Cont. Apps., Anchorage AK. Sept 2000,PP.467-72.

[42] Bulusu et al., "Scalable Coordination for Wireless Sensor Networks: Self Configuring Localization Systems," ISCTA 2001, Ambleside, U.K, July 2001. 